\title{
Ambispective study on Mac Donald suturing in pregnant ladies with cervical incompetence in Dhulikhel Hospital
}

\author{
Tamrakar SR ${ }^{1}$, Chawla $\mathrm{CD}^{2}$
}

${ }^{1,2}$ Department of Gynecology \& Obstetrics,
Kathmandu University Hospital, Kavre, Nepal.

\section{Corresponding author}

Dr Suman Raj Tamrakar

Department of Obstetrics and Gynaecology,

Dhulikhel Hospital

Kathmandu University Hospital, Kavre, Nepal

Email: drsuman537@yahoo.com

Kathmandu Univ Med J 2010;8(31):321-24

\section{ABSTRACT \\ Background}

Cervical incompetence is one of the main contributors to repeated pregnancy loss, accounting for approximately $25 \%$ of the cases. Typically it results in progressive cervical dilatation, leading to a painless second- or early-third-trimester abortion.

\section{Objectives}

The main objective of the study was to explore the benefit from cervical cerclage in pregnant women with anatomical cervical incompetence

\section{Methods}

In a review of the operation and labour registers from January 2006 till January 2010, a total of 38 cervical cerclage procedures were performed at Dhulikhel Hospital (DH). In the study caste, parity, gestational age, diagnostic criteria, postoperative complications and pregnancy outcomes of the cases were analyzed.

\section{Results}

Two of the 38 cases didn't come for delivery at Dhulikhel Hospital (Kathmandu University Teaching Hospital). Four women haven't delivered at the time of data analysis. So pregnancy outcomes were analyzed among 32 cases while rests of the variables were analyzed among 38 cases. Of them 18 cases $(47 \%)$ were Brahmin, 22 cases $(58 \%)$ were between 20-25 years old and 32 cases (84\%) were from Kavre district.

All cases were booked cases (they had antenatal care in the hospital) and 14 patients (37\%) were third gravida. Most cases had 2 to 4 antenatal visits prior to suturing. Two cases were diagnosed with a bicornuate uterus. 21 cases $(55 \%)$ had a previous history of at least one dilatation and evacuation.

33 cases $(87 \%)$ were diagnosed with cervical incompetence clinically and confirmed by ultrasound. The remaining $13 \%$ were assessed, in the absence of a history of midtrimester abortion, of having a high suspicion of cervical incompetence after midtrimester scan with measurement of cervical length. In 18 cases (47\%), cervical cerclage were done at 15 to 20 weeks of gestation. The postoperative period was uneventful in all 38 cases.

All cases (32) delivered in DH were assisted by consultant obstetricians. 19 out 32 cases (59\%) were delivered vaginally at term.

\section{Conclusions}

38 cases were included in the study. Pregnancy outcomes were analyzed among 32 cases while rests of the variables were analyzed among 38 cases. 31 out 32 cases were delivered with good foetal weight. It clearly shows pregnant women with anatomical cervical incompetence were benefitted from cervical cerclage.

The authors recommend an early trans vaginal scan in any patient with a history of mid trimester abortion or preterm labour. The cervical cerclage procedure therefore should be available more widely to benefit those patients with proven or strongly suspected cervical incompetence.

\section{Key Words}

cervical incompetence, cervical encirclage, trans vaginal scan 


\section{INTRODUCTION}

The frequency of clinically recognized spontaneous abortion in the general population has been estimated to range between $15-20 \% .{ }^{1}$ Approximately $80 \%$ of spontaneous abortions occur in first trimester. The incidence of abortions decreases with increasing gestational age. Though chromosomal abnormalities and genital infections are responsible for many first trimester abortions, cervical incompetence is one of the important causes of mid-trimester and recurrent abortions.

A history of second or early third trimester fetal loss, after painless dilatation of the cervix, prolapse or rupture of the membranes, and expulsion of a live fetus despite minimal uterine activity, is characteristic for cervical insufficiency. ${ }^{2}$

Cervical incompetence (CI) is considered as a defect of the cervical stroma that takes to the inability of the uterine neck to retain an intra-uterine pregnancy until the viability of the fetus, with an incidence from $0.05 \%$ to $1 \%$ of all the pregnancies. ${ }^{3}$

Cervical incompetence is one of the main contributors to repeated pregnancy loss, accounting for approximately $25 \%$ of the cases. Typically it results in progressive cervical dilatation, leading to a painless second- or early-thirdtrimester abortion. ${ }^{4}$

The diagnosis of cervical insufficiency is notoriously difficult to make, and is usually a retrospective one based on a history of recurrent second trimester loss (or early preterm delivery) following painless cervical dilatation in the absence of contractions, bleeding, or other causes of recurrent pregnancy loss. ${ }^{5}$

Cervical incompetence can be diagnosed from precise obstetric history, physical examination and with different technical aids like ultrasound, dilators and catheter balloons. In this regard, trans vaginal scan (TVS) is the most appropriate tool for measuring the cervical length. We can perform prophylactic cervical encirclage at 13 to 16 weeks in cases with cervical incompetence.

Cervical cerclage still represents the only surgical treatment for cervical incompetence. In the last 20 years (1971-1990) we performed $272 \mathrm{Mac}$ Donald cervical cerclages. In 16 cases the outcome of pregnancy is unknown; 198 women (73.3\%) subsequently delivered healthy infants later than 37 weeks' gestation or weighing more than $2500 \mathrm{~g}{ }^{6}$

Uterine cervical incompetence is one of the most common cause of habitual abortion in the second trimester of pregnancy and premature delivery; cervical cerclage still represents the only surgical treatment for cervical incompetence. ${ }^{6}$

In spite of the fact that numerous procedures have been advocated as treatment, the Mac Donald purse string closure technique is by far the most simple and effective corrective surgical technique, yielding an overwhelming number of successful pregnancies. ${ }^{4}$

Currently the Mac Donald suturing technique is the mostly practiced method among different trans-vaginal methods.

Hence fetal loss is a painful experience. In such cases the risk of recurrence is high, and a policy of prophylactic cerclage may be safer than one of serial cervical length measurements followed by cerclage, tocolysis and bed rest in case of cervical shortening or dilatation. ${ }^{2}$

\section{METHODS}

To analyze caste, parity, gestational age, diagnostic criteria, postnatal complications,pregnancy outcomes of the cases with cervical incompetence and explore the benefit from cervical cerclage.

In an ambispective study of the operation and labour registers from January 2006 till January 2010, a total of 38 cervical cerclage procedures were performed at Dhulikhel Hospital (Kathmandu University Hospital). Clinical variables evaluated included gestational age at cerclage, gestational age at delivery, cervical dilation at presentation, and presence or absence of bulging membranes on admission. Presence or absence of clinical symptoms at presentation or historic risk factors for incompetent cervix was also noted. Cervical length was measured along with anomaly scan at 20 weeks in all antenatal cases. All the patients with proven or strongly suspected cervical incompetence were included in the study. In all the cases with cervical incompetence, a standard protocol has been followed. The data was compiled, analyzed and interpreted.

\section{RESULTS}

Two of the 38 cases didn't come for delivery at Dhulikhel Hospital (KUH). Four women haven't delivered at the time of data analysis. So pregnancy outcomes were analyzed among 32 cases while rests of the variables were analyzed among 38 cases. Of them 18 patients (47\%) were Brahmin, followed by those of Chhetri, Newar, Lower caste, Tamang and others. 22 cases (58\%) were between $20-25$ years, 8 cases $(21 \%)$ were of $25-30$ years, 6 cases $(16 \%)$ were of 15-20 years and 2 cases (5\%) were of 30-35 years old. 32 patients (84\%) were from Kavre district followed by rest from Sindhupalchowk and then from Kathmandu valley.

All cases were booked cases (they had antenatal care in the hospital). Most cases had 2 to 4 antenatal visits prior to suturing. Two cases were diagnosed with a bicornuate uterus. 21 cases (55\%) had a previous history of at least one dilatation and evacuation. 14 patients (37\%) were third gravid (Figure 1). 
Figure 1. Parity of the cases underwent Mac Donald suturing

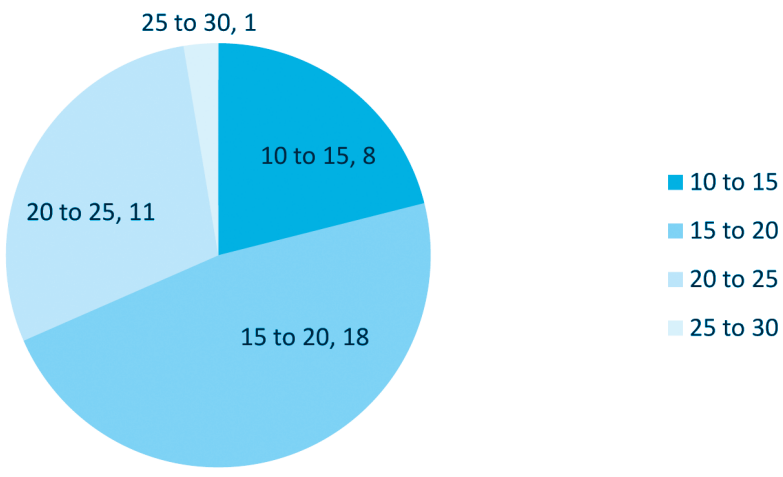

33 cases (87\%) were diagnosed to have cervical incompetence clinically and confirmed by ultrasound. The remaining 5 cases (13\%) were assessed, in the absence of a history of mid-trimester abortion, of having a high suspicion of cervical incompetence on the basis of a TVS measured cervical length less than $2.5 \mathrm{~cm}$ at a 20 week scan.

In 18 cases (47\%), cervical cerclage were done at 15 to 20 weeks of gestation (Figure 2). The postoperative period was uneventful in 38 cases. No one had any postnatal complications like bleeding or watery discharge per vaginum, pain lower abdomen etc.

\section{Figure 2. Period of gestation distribution of cases} underwent Mac Donald suturing

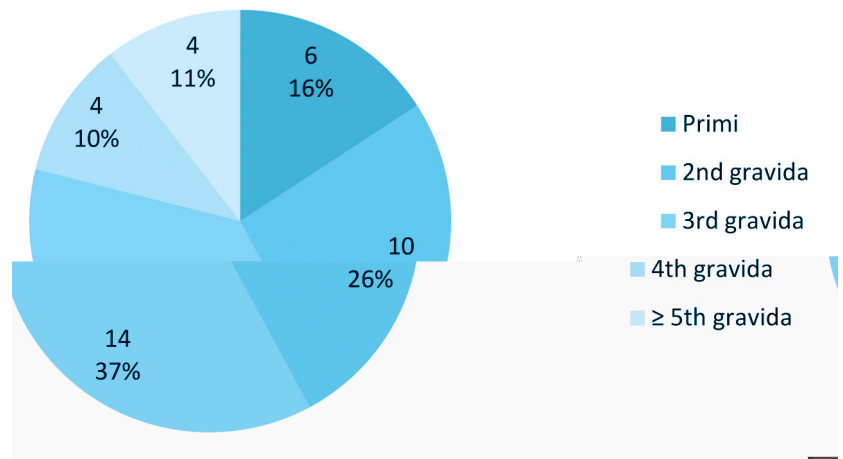

19 out 32 cases (59\%) were delivered vaginally at term. Remaining twelve cases (37\%) were delivered via caesarean for different indications like foetal distress, non progress of labour, malpresentation etc. Of them three cases were delivered prematurely. Two women diagnosed with bicornuate uterus. One woman had cervical cerclage and delivered through caesarean at term. But another woman aborted (a foetus weighing 800 gram) at 19 weeks in spite of the insertion of the cervical circlage and vigilant hospital based obstetrical care.

All cases (32) delivered in DH were assisted by consultant obstetricians.

\section{DISCUSSION}

Cervical incompetence is one of the main contributors to repeated pregnancy loss. In most instances cervical incompetence is the result of previous obstetric or gynecologic trauma; a congenital etiology is unusual. Despite the various reported diagnostic procedures, the obstetric history and frequent visual and digital examinations during pregnancy remain the most important factors in arriving at the correct diagnosis. ${ }^{4}$ In $\mathrm{DH}, 33$ cases $(87 \%)$ were diagnosed with cervical incompetence clinically and confirmed by ultrasound. 21 cases (55\%) had a previous history of at least one dilatation and evacuation.

Cervical incompetence remains a controversial area in obstetric management. The development of transvaginal sonography has resulted in a clearer image of the internal cervical os, a development which may indirectly have beneficial effects on outcome. ${ }^{7}$ In $\mathrm{DH}, 5$ cases (13\%) with the absence of a history of mid-trimester abortion, of having a high suspicion of cervical incompetence on the basis of a TVS measured cervical length less than $2.5 \mathrm{~cm}$ at a 20 week scan.

In a study by Nava et al 36 patients were included, with $30.1+/-4.2(\mathrm{M}+/-\mathrm{SD})$ year-old age; the cerclage was performed in the second trimester, in order to extend the pregnancy with an average of $18.1+/-6.5$ weeks until the moment of the interruption of the pregnancy. The $61.1 \%$ arrived to the term gestation and in 23 of the cases (63.8\%) were born with more than $2500 \mathrm{~g}$ of weight. The main indicators of success are the cerclage application to smaller gestational age, into the second and third gestation, as well as the presence of the smallest number of complicated obstetric events. ${ }^{3}$

In $\mathrm{DH}, 38$ cases ( $58 \%$ ) were between $20-25$ years old. The postoperative period was uneventful in all 38 cases. 19 out 32 cases (59\%) were delivered vaginally at term with good foetal weight.

In a study of 275 cases, they have analysed the efficacy of cervical cerclage in the prevention of miscarriage in patients with uterine malformations. The data indicate that cervical cerclage is effective in preventing miscarriages, prevalently in those pregnancies bearing uterine malformations with simultaneous cervical incompetence. ${ }^{8}$ In $\mathrm{DH}$, two cases were diagnosed with a bicornuate uterus.

The records of seventy-four patients who had had cervical cerclage procedures were surveyed. Without suture placement, these patients would have had a fetal salvage rate of $23 \%$. In the 60 patients, 6 pregnancies terminated between the 28th and the 36th week of gestation, and 54 pregnancies were carried beyond the 36th week; all of these infants survived. The salvage rate after the cerclage procedure was $81.8 \%$. Eight caesarean sections were performed (13.3\%) for various reasons. ${ }^{9}$ In DH 19 out 32 
cases $(59 \%)$ were delivered vaginally at term.. Remaining twelve cases $(37 \%)$ were delivered via caesarean for different indications like foetal distress, non progress of labour, malpresentations etc. Of them three cases were delivered prematurely.

\section{CONCLUSIONS}

38 cases were included in the study. Two of them didn't come for delivery at DH. Four women haven't delivered at the time of data analysis. So pregnancy outcomes were analyzed among 32 cases while rests of the variables were analyzed among 38 cases. 31 out 32 cases were delivered with good foetal weight. The pregnancy outcomes were quite encouraging for both patients and clinicians. It clearly shows pregnant women with anatomical cervical incompetence were benefitted from cervical cerclage.

The authors recommend an early TVS in any patient with a history of mid trimester abortion or preterm labour otherwise cervical length should be measured along with anomaly scan at 20 weeks in all antenatal cases. In such cases a cervical length of less than $2.5 \mathrm{~cm}$ should be considered for cervical cerclage. This procedure therefore should be available more widely to benefit those patients with proven or strongly suspected cervical incompetence.

\section{REFERENCES}

1. Rock JA, Zacur HA, The clinical management of repeated early pregnancy wastage. $F$ 1983;39:123-40.

2. Lotgering FK., Clinical aspects of cervical insufficiency. $B M C P$ a c $C$ 囚b 2007; 7:S17

3. Nava Flores J, Morales Toia ME, Veloz Martínez G, Hernández-Valencia M. Efficacy of elective cerclage in patients with cervical incompetence and associated risk factors. $G \quad c \otimes O b \mathbb{B} M 2003 ; 71: 356-62$.

4. Ansari AH, Reynolds RA., A review of cervical incompetence. JR M 1987;32:161-71.

5. Simcox R, Shennan A., Cervical cerclage: a review. I S 2007;5:205-9.

6. D’Addato F, Malagnino F, Repinto A, Mocchia M, Andreoli C., Cervix cerclage: A 20-year case load. $M$ a $\quad$ a $\quad c$ オ 1992;44:313-6.

7. Chung TK, Haines CJ, Kong D, Woo WK, Rogers MS., Transvaginal sonography in the diagnosis and management of cervical incompetence. $G \quad c \quad \nabla O b$ 极I 1993; 36:5961.

8. Surico N, Ribaldone R, Arnulfo A, Baj G., Uterine malformations and pregnancy losses: is cervical cerclage

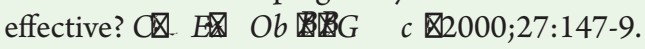

9. Schwartz RP, Chatwani A, Sullivan P., Cervical cerclage: A review of 74 cases. JR $\quad M \quad$ 1984;29:103-6. 